Elshad A. Baliyev', Kamran A. Abilov'

DOI: 10.25045/jpit.v08.i2.05

Azerbaijan Technical University, Baku, Azerbaijan

1b.elsad@list.ru, ${ }^{2}$ kamran_a72@mail.ru

\title{
ANALYSIS OF DATA TRANSMISSION ENVIRONMENT IN OPEN SYSTEMS AND DEVELOPING THE GRAPH MODEL
}

The article analyzes the network environment in computer networking technologies. To this end, the study shows the logical channels between processes, the direction of the flow of information between the communication node, the logical structure of the transport network, investigated packet transmission medium. Based on the abovementioned and the OSI models, graph-model relationship of open systems is developed.

Keywords: The OSI reference model, network layers, network protocols, communication node, package.

\section{Introduction}

At present, modern information technologies are highly developing. As a result, today there is no any industry, scientific-technical, medical, oil-gas, education and other areas in which computers and computer networks would not be widely used [1-3].

In such circumstances, it is important to provide reliable and high quality services to network users. To achieve this goal, the development of models for effective transmission of data over computer networks is crucial.

Obviously, the data transmission medium on the computer networks, especially on the Internet, is based on ISO/OSI (International Standardization Organization / Open System Interconnection) model. International Standardization Organization has developed a base model, which is an open systems reference model, for the data delivery on the networks consisting of heterogonous devices and software $[4,5]$. The article aims to analyze the data transmission medium in open systems and to develop the graph model.

The ISO/OSI model explains the stages and rules for data transmission in different network mediums during the connection session. The key elements of the model include the management levels, application processes, and physical connection tools (Figure 1).

Each level of the reference model OSI performs certain tasks in the data transmission via the network. The base reference model is considered to be the key model for the development of the network protocols. The OSI model divides the communication functions by seven network levels, each of which serves to different parts of the interconnection process of the open systems [6,7].

The OSI model only explains the interconnected system tools without touching the end users' attachments. Attachments perform the specific protocols of interconnections by applying to system tools. If the attachments take on the functions of some top levels of OSI model, then it directly applies to the system tools that run the functions of the remaining low levels of OSI model for data sharing.

The reference model OSI shown in Figure 1 can be divided into two different models: a horizontal model based on the protocols that provide a mechanism for interactions between the processes and programs on different machines, and a vertical model based on the services of the adjacent levels in the same machine providing each other.

Each level of the sender interconnects with the host with the same levels, and it seems that it is directly related. Such a relationship is called a logical or virtual connection. In fact, mutual interactions are carried out at a mixed inter-level within a computer $[4,8]$.

The information in a sending computer passes through the all levels. Then, it is transmitted to the host computer through the physical environment and goes straight through all the levels again. This process is performed until the same level of the sender.

The horizontal model requires a common protocol for data sharing. In the vertical model, the neighboring levels perform the data sharing using the application interface. 
The data is divided into packets before being sent to the network. The packet is an inter-station transmitted information unit on the network. When the data is sent, the packet is transmitted consistently through the all levels of the software. At each level, the managing information (headings and endings) of that level is added to the package for the successful data transmission via the network $[5,6]$.

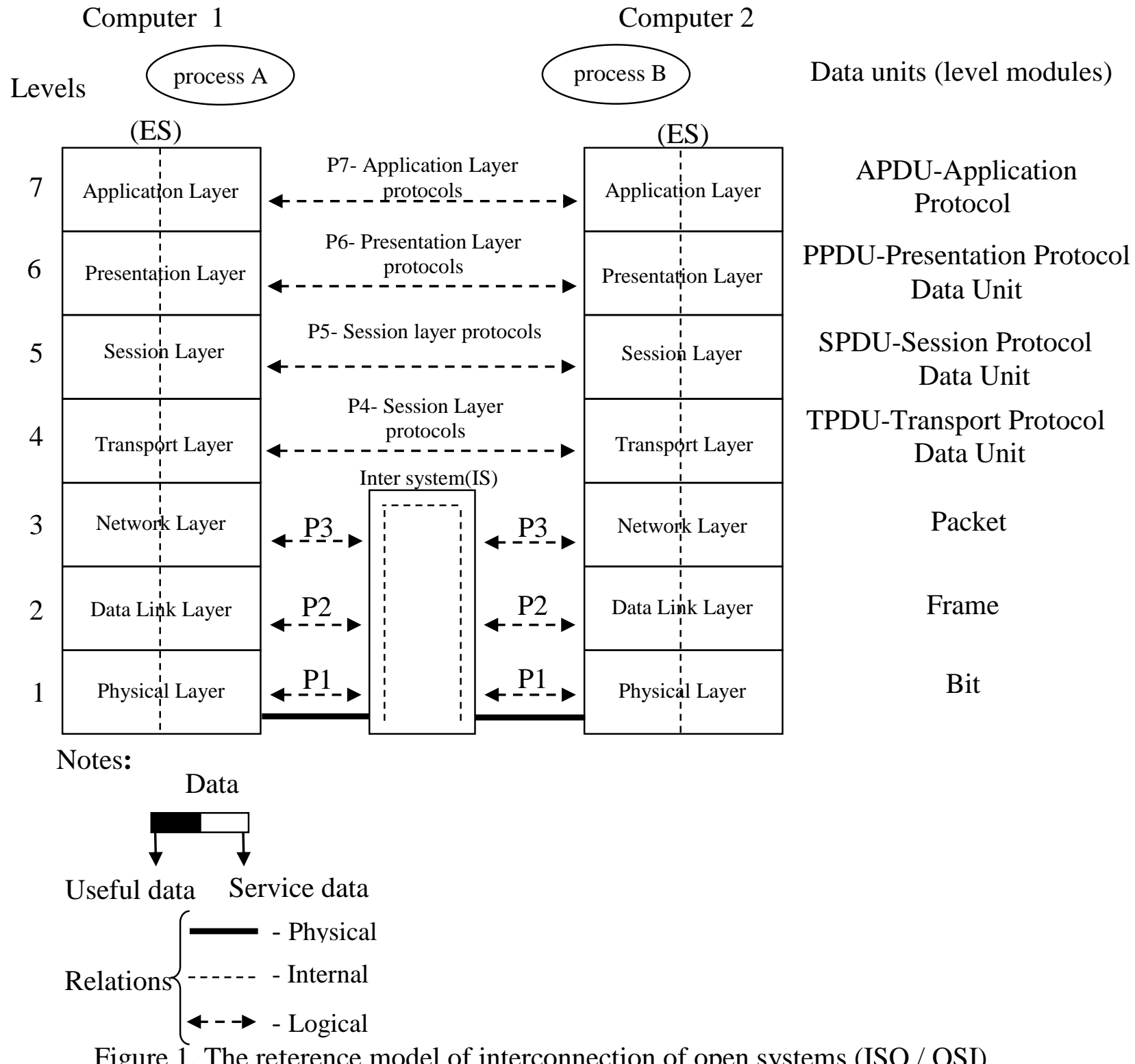

Figure 1. The reterence model of interconnection of open systems (ISO / OSI)

Once the packet reaches the application level, all managing information is removed from the package (i.e, deleted) and the data is brought to its original status.

Thus, each level of the model performs its own function. The higher the level, the more difficult it will be solved.

Based on this commonly provided methodological principle, let us briefly clarify the levels of the reference model OSI.

\section{The analysis of the tasks and functions of the reference model OSI by the management levels}

It is known that the physical layer of the reference model OSI transmits the bits through the twisted wire pairs, coaxial cables, optical fiber cables and so forth. At this level, the physical data transmission medium, that is release line, hinge protection, wave resistance and so on are taken into account. The characteristics of the electrical signals of the discrete information - the voltage and current of the transmitted signals, the encoding type, the transmission speed, and etc are determined. The 
physical level function is implemented by all devices connected to the network. In the computer, its function is implemented by adapters or consecutive ports. In this case, some networks do not take into account the busy physical medium of the transmission, since the connection lines are changed and used in alternately inter-connected computer pairs. At this level, data distortion varies within $10^{-4}-10^{-6}$ errors/bit. Therefore, one of the key issues of the Data link layer is to check the calls (applications) to the transmission medium. Another issue of this level is the adjustment and detection of errors. For these purposes, the bits are grouped in the sets - so called frames. The data link level adjusts the errors occurred in the transmission of each frame and enters them by connecting the individual bits sequence to the beginning and end of each frame, to separate them from one another. It calculates the control total by processing all the bytes of the frame by a certain method and adds the result to the frame. When the frame passes through the network, it calculates control total of the received data again and compares the result with the control total in the frame. If they coincide, the frame is considered to be correct and accepted. Otherwise, the errors are recorded. The errors detected in such cases are eliminated by the re-transmission of the damaged frames. The amount of distortion at the data link level varies within $10^{-8}-10^{-9}$ errors/bit.

The Network level provides the creation of a single transport system that connects several networks with different data transmission principles. Networks are integrated with a specific device called a router. The router collects information about network interconnection topologies and transmits the network-level packets to a certain address. To deliver packets sent from the network level to the receiver, it goes through transit passes via several interconnected nodes and performs the optimal route selection and the implementation of different protocols. Thus, the transport level has a crucial importance as the next stage of the inter-level interconnectedness..

The main function of the Transport Layer is to receive the data from the session level, if necessary, to divide it into smaller parts (packets), and pass it to the network level ensuring that it is correctly delivered to the destination. In addition, when all abovementioned are executed, the higher levels must be isolated so that any change in the apparatus residuals does not affect the data to be sent. The transport level determines the types of the service offered to the session level and defines the use of the channel between the two nodes that are protected from errors and the direct delivery from the source to the users.

The Session layer is used to create a communication session of different computers. In this case, various types of services are provided: dialogue management, marker management and synchronization.

Particular attention is paid to the data transmission tracking in the Dialogue Management Service.

The marker management service is envisioned to prevent the simultaneous execution of the critical operations together with several systems.

After the errors are eliminated by putting the service marks into the long data in the Synchronization service, the data transmission continues from the breaking point.

Unlike the low levels, the Presentation layer is of particular importance for more accurate transmission of bits and bytes. This level actually deals with the syntax and semantics of transmitted information. In order to make computers to be able to share different presentation data, it is important to convert the data format to each other by transferring it to the network. The presentation level engages in the mentioned transformations by introducing the data determination capability and the changes in the structure of higher levels.

Finally, the Application layer performs the process of processing and consists of a set of frequently-used protocols needed to users (Figure 1) [4, 5].

To deliver high level quality service to the users taking into account all abovementioned, in order to develop the graph model of transporting the multiple interprocess data available in the transport level of the reference model OSI through the logical channel, as well as the communication nodes to the required process (the end host machine), the logic channel description, the interports data transmission via the logical channels and logical structures of the transport network should be reviewed. 


\section{The analysis of data transmission medium in open systems}

As with the real computing networks, the physical channel management is not carried out with the machines, but with the data transfer equipment connected to it. The information channel management program is often implemented with the same equipment. As mentioned above, the ports are located between the data transfer management level and the processes. The dotted lines between the different host machines (host-user programs, that is, the machines running the applications and geographically remotely located and connected to the subnets) and the terminal machine ports show the logical channels (Figure 2). One of these channels is the logical channel linking the process A of the first host machine to the process B of the second host machine.

Apparently, this channel goes from the port of the first host machine to the port of the second host machine via the physical channels and connection nodes (communication machine). Depending on the network condition and its loading, the logical channel may pass not only through the route presented in the figure, but all the possible routes of the network. The data transmission from one port to another is related to the fact that the network repeatedly passes through the different levels of the software structure. The data transmission from the process A to the process B via logical channel can be described as in Figure 3.

Similarly, the data can be transmitted in networks by any routes of the numerous logical channels and communication nodes within many other processes.

It should also be noted that the logical channel is canceled after the end of the inter-process communication session.

Along with those listed, the transport level is a cross-border frontier zone, and its objective is to transmit the data among the session-level objects. The session level is referred to as transport users and the transport level provides these users with the transport connections, which are the ports of the network. In this case, one or more transport connections are identified between a pair of transport users.

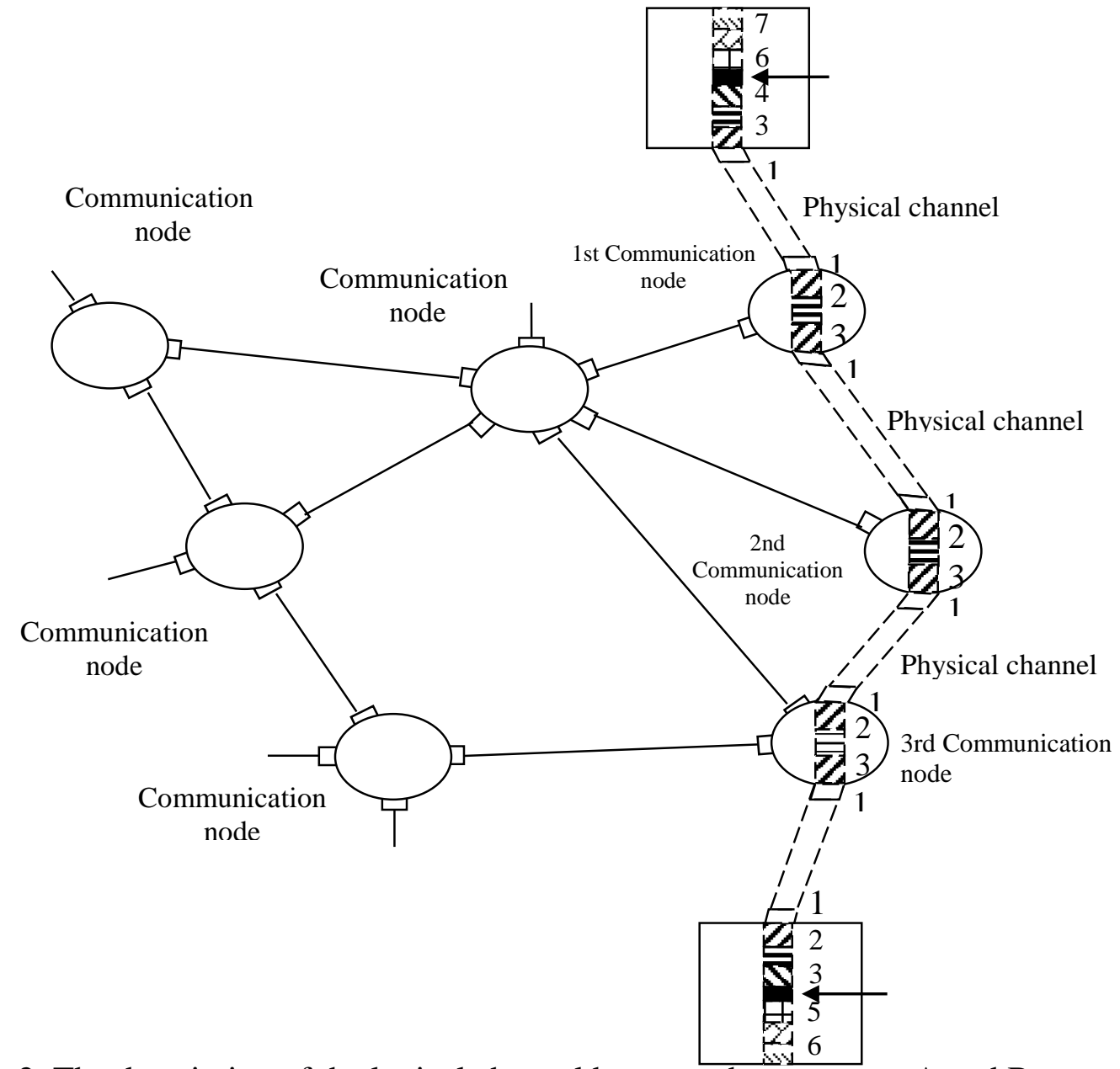

Figure 2. The description of the logical channel between the processes A and B 


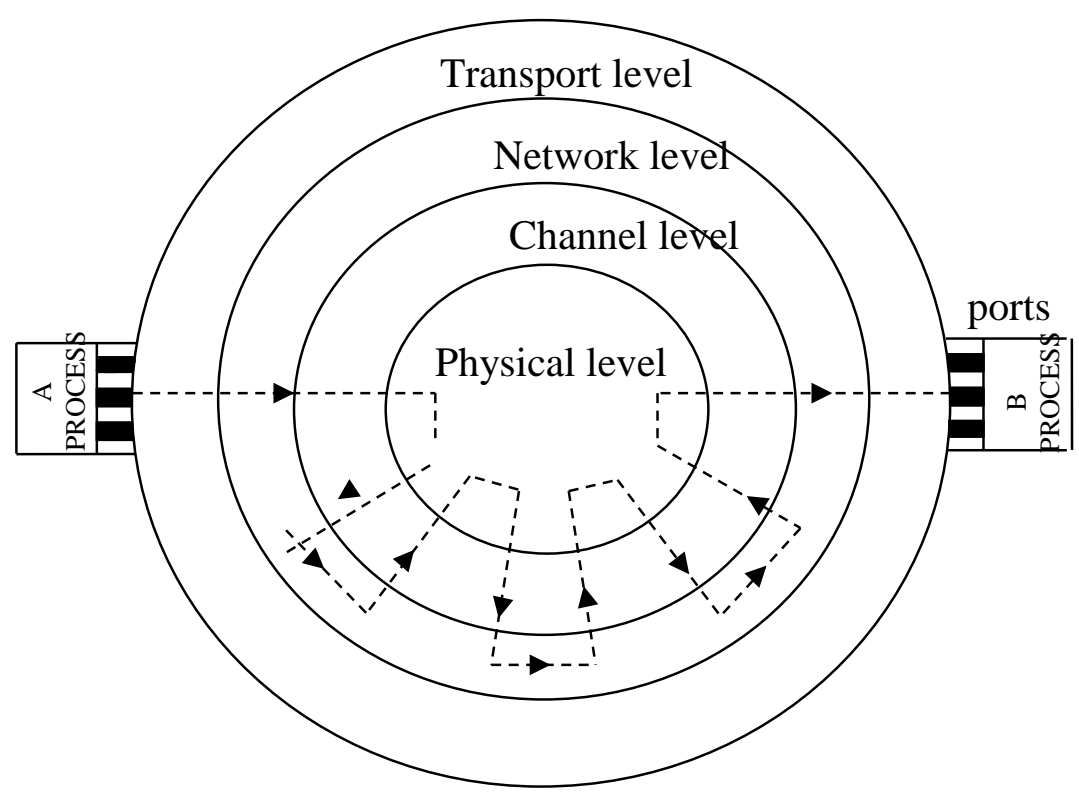

Figure 3. Data movement through the inter-process logical channel

The transport service is provided by the transport services of a highly rated dataset.

The performance of the transport level is determined by its protocols. It can be noted that the transport level protocols determine the data transmission across the entire transport network from one port to another. Taking into account the listed descriptions (Figure 1 and Figure 2) and all the above-mentioned, the logical structure of the transport network can be described as in Figure 4 .

The logical system is a transport channel that provides the data transmission between the two blended computing machines connected with the physical channel. This transmission is explained by two low level protocols of the software structure of the computing networks as defined by the management of the physical channel and data channel. A logical system that locates at a higher level and enables the transport network building, is reviewed, which is explained by the transmission and network management protocols.

As seen from Figure 4, the transport network consists of two types of interconnected logical modules: communication and transport.

Transport modules provide the transport network access, manages the network, and only the communication modules perform the data routing.

Here, the communication modules, which are a part of the transport network and interconnected with the superior channels, are of great importance. It is called a communication or data transmission network (DTN).

In the transport network, the packets are transmitted from one logical module to another. The packet is placed in the data frame before being transmitted, and after being transmitted it is removed from the frame. Thus, the packet moves through the transport module router and passes through the communication module and the physical channel, and then is transmitted to the address receiving the transport module. For example, the packets are sent from any port to another (picture 4). There are several routes for this transmission in the transport network. Here, any packet $n$ is transmitted through the communication modules $K, L, M$. The packet $n$ is packed into the frame $a$, and sent to the communication module $k$. Where the packet $n$ is removed from the frame $a$, the errors are checked and the destination address of the packet is analyzed. Accordingly, the address of one of the communication modules is selected and the packet $n$ is transmitted. Subsequently, it is placed in the frame $f$ and transferred to the communication module $L$. Where the packet $n$ is removed from the frame again, checked and forwarded to the communication module $M$ according to the destination address (but as packed in the frame $t$ ). 


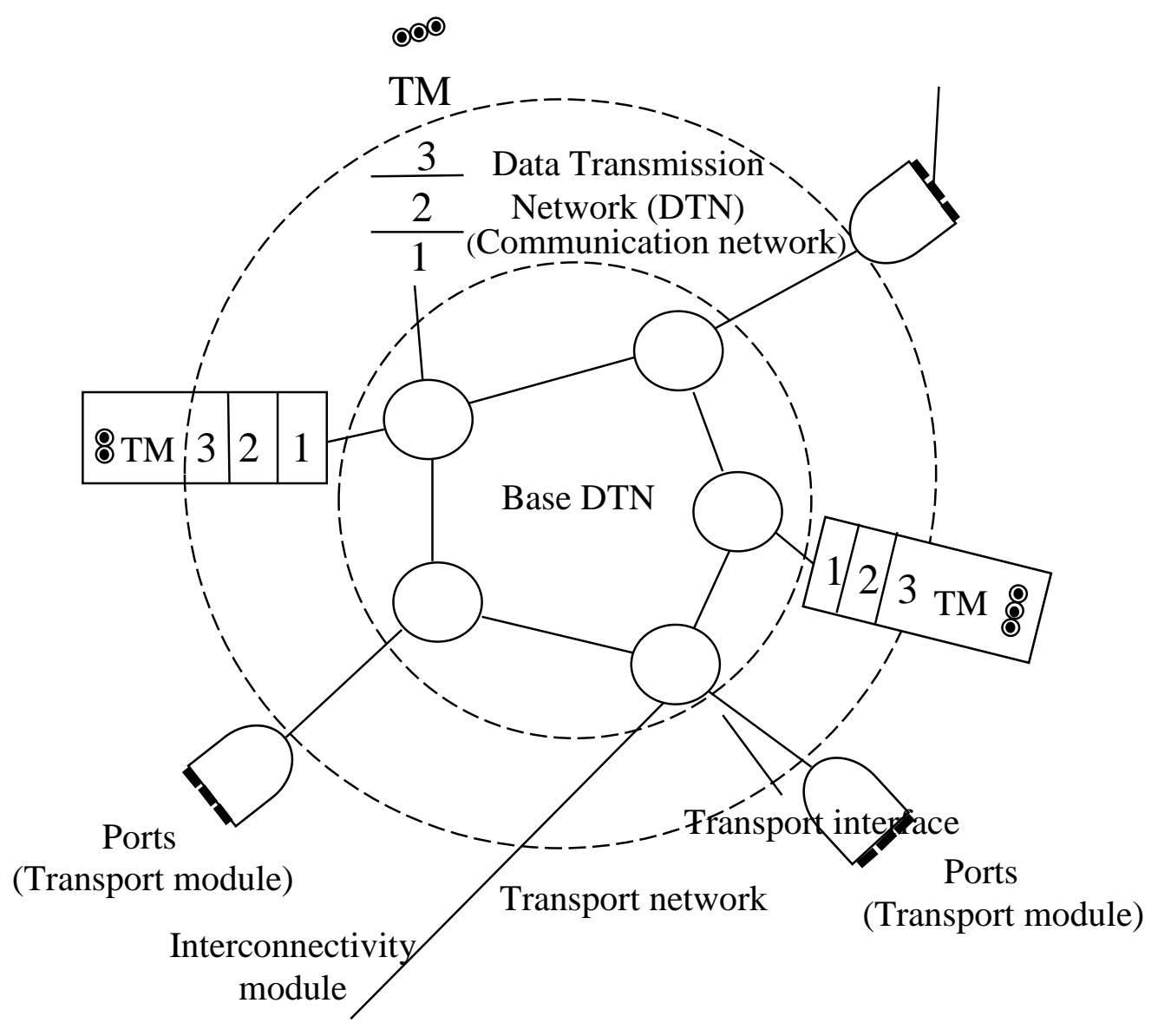

(Communication module)

Figure 4. Logical structure of the transport network

The aforementioned is carried out once again in the communication module $M$, then delivered to the packet $n$ placed in the frame $s$ and finally to the port of the transport module.

Thus, it is possible to transfer not only to the user data, but also the packets containing the management information of the transport network.

Evidently, computing networks protocols are relatively not dependent on one another $[4,5$, 7]. This enables to change the software structure of a level without changing the software structure of another one. This, in turn, enables to place the packets on different types of frames when transferring them via the computing network. The protocol HDLC (High Level Data Link Control) is highly effective and common. There are numerous information channels that use only the channel management protocol. Therefore, it is not accidental that the HDLC frames are placed in some channels, while the channels management protocols are placed in the other one. Thus, the transport network must be capable of solving many different requirements. They may include $[8,9]$ :

1) transmission of the size-limited packets (not exceeding 2000 bits);

2) the packages' transmission delay shall be small (less than $0,5 \mathrm{sec}$ );

3) low level errors are acceptable $\left(10^{-10}\right.$ errors/bit);

4) the packet storage (not more than $10^{-4}$ errors/packets);

5) inclusion of data of any bit into the packet is available, i.e., the transparency for all packages shall be provided. 


\section{Developing the graph model of open systems}

All these requirements define the structure of the transport network consisting of the transport channels of an independently functioning logic systems. Thus, the transmission and network management levels provide the data distribution across the network.

The graph model of the open systems can be presented as in Figure 5, if taking into account the data movement through the inter-processes logical channel, the logical structure of the transport network, high quality service class with the transport level, as well as the networkdependent and independent inter-level operability of the reference model OSI [10].

Based on the OSI reference model and the graph model of the interconnection of the described open systems, it is possible to develop a graph model of other existing reference models (TPC/IP, IPX/SPX, hybrid etc.) $[4,7,11]$. At the same time, there is a need for more detailed study of the graph-model of the interconnection of the described open systems in the open systems and the operation and logical relations of the network, transport and application levels, basing on TCP/IP reference model [7, 11]. A number of other reference models prove that there is a need for a more thorough investigation to achieve rather advanced reference model of open systems.

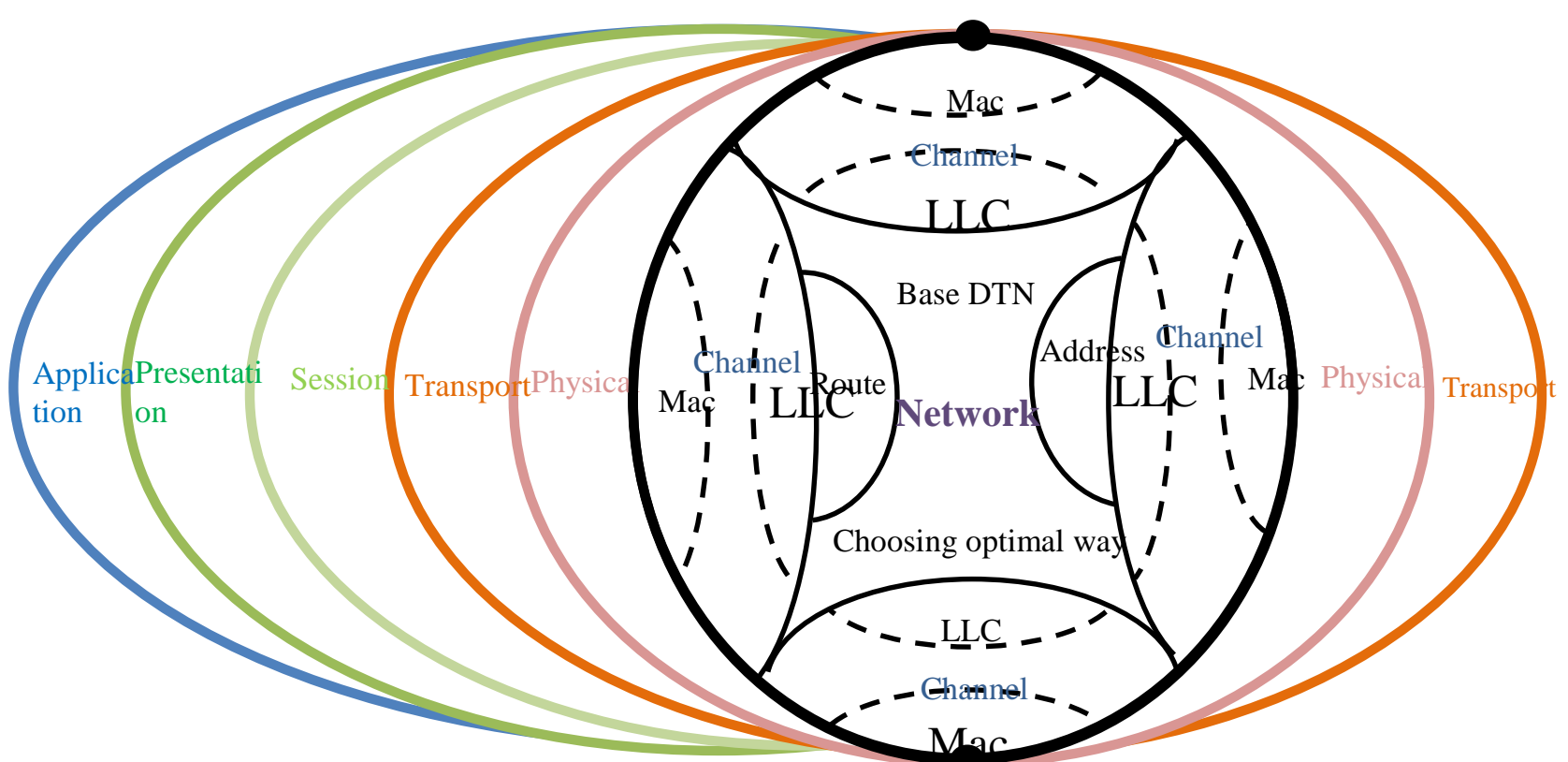

Figure 5. The graph model of the interconnection of the open systems

\section{Conclusion}

The paper analyzed the tasks and functions of the managing levels of the reference model OSI, and the data transmission medium in the open systems and developing the graph model of the open system. We can conclude that one of the important functions of the transport level, which is directly related to any of the existing reference models OSI, is providing more reliable and better quality services to the users. To achieve these goals, the transport level is called a transport object, as it uses the software along with the equipment performing the network-level implementations. The transport object is loaded with the network attachments and located in the separate user processes, operating system core or on the network interface board. As a result, three horizontal interfaces emerge: network, transport and application. These urge the researchers to define the logical relationships of the network, transport and application levels of the open systems in details, and ultimately to achieve a more efficient and promising model. 


\section{References}

1. Alquliyev R.M., Imamverdiyev Y.M. Conceptual BIG DATA architecture for oil and gas industry // Problems of Information Technologies, 2017, No1, pp.3-14.

2. Alakbarov R.G., Alakbarov O.R. Mobile computing clouds: current state, architecture and problems // Problems of information technology, 2017, No1, pp.42-52.

3. Aliquliyev R.M., Niftaliyeva G.Y. Current status, problems and prospects of e-government analysis technologies // Problems of Information Technologies, 2017, No1, pp.59-70.

4. Tanenbaum E. Computer networks. 4th ed. St. Petersburg: Peter, 2003, 992 p.

5. Olifer V.G. et al.. Computer networks. Principles, technologies, protocols. St. Petersburg: Peter, 2003, 864 p.

6. Faith C. TCP/IP. Architecture, protocols, implementation, M.: Williams, 2003, 424 p.

7. Shaposhnikov I. Computer networks. Principles, technologies, protocols, St. Petersburg: Peter, 2006, 304 p.

8. Jafarov Z.A. Analysis of addressing systems in telecommunication networks // Problems of information technologies, 2014, No1, pp.69-80.

9. Maximov N.V. et al. Computer networks, M.: Forum, 2010, 464 p.

10. Baliyev E.A., Abilov A.K. Development of graph model of the open systems / Proceedings of the International scientific-technical conference dedicated to the current state and development prospects of ICT, Baku, AzTU, 23-25 November, 2016.

11. Parker T. et al. TCP/IP. For professionals. 3rd ed. St. Petersburg: Peter, 2004, 859 p. 